\title{
Post Pregnancy Severe Spinal Osteoporosis with Multiple Vertebral Fractures and Kyphoscoliosis in a Multigravida: A Rare Case with Management
}

\author{
Shailesh Hadgaonkar, Kunal Chandrakant Shah, Hrutvij Bhatt, Ashok Shyam, Parag Sancheti \\ Sancheti Institute for Orthopaedics and Rehabilitation, Sancheti Hospital, Pune, India
}

Osteoporosis associated with pregnancy and lactation is a less commonly known condition and often overlooked. The prevalence, exact aetiology and its pathogenesis are unknown. It is commonly seen in first three months after delivery in primigravida. It is often undiagnosed because of it not suspected $\mathrm{n}$ and $\mathrm{X}$-rays and densitometry are avoided if possible during pregnancy and lactation. If missed, it can lead to osteoporotic fractures and disability. In this paper, we report a case of a 24-year-old multigravida 4 months after pregnancy with multiple vertebral compression fractures and kyphoscoliosis. Her metabolic workup was normal but bone densitometry revealed severe osteoporosis of the dorso-lumbar spine. Immediate weaning and antiresorptives like bisphosphonates and teriparatide are used as first line drugs to manage postpartum spinal osteoporosis. Our patient presented at 4 month lactation and did not want to wean her infant, so she was treated with total contact orthosis and took vitamin D and calcium. The pain was relieved within 3 months but there was no improvement in bone density. After eight months when the infant was weaned, she was treated with teriparatide. After one year of teriparatide therapy, there were no new fractures and densitometry scores improved.

Keywords: Post-pregnancy; Osteoporosis; Spinal fractures; Teriparatide

\section{Introduction}

Pregnancy-associated osteoporosis described by Nordin and Roper in 1955 is a rare condition that results in back pain during pregnancy and the postpartum period, and causes significant disability [1]. There are four types of osteoporosis associated with pregnancy described in literature, viz idiopathic osteoporosis, transient osteoporosis of hip, post pregnancy spinal osteoporosis (PPSO) and lactation associated osteoporosis [1]. PPSO presents as a rare cause of back pain in late pregnancy and during lactation [2]. If missed or untreated, it increases the risk of a patho- logical vertebral compression fracture and subsequent deformity [3]. The magnitude of problem is underestimated and diagnosis is often missed because of the rarity of the disease, avoidance of radiographs and densitometry during pregnancy and back pain is often attributed to other factors during pregnancy [2].

Our aim is to report this unique case of postpartum severe spinal osteoporosis with multiple vertebral fractures and kyphoscoliosis in a lactating multigravida female with normal first delivery to highlight the diagnostic challenges, socio-economic impact and emphasis on the role of newer medical management.

Received Jul 12, 2014; Revised Jul 29, 2014; Accepted Aug 3, 2014

Corresponding author: Kunal Chandrakant Shah

Sancheti Institute for Orthopaedics and Rehabilitation, Sancheti Hospital, 16, Shivajinagar, Pune 411005, India

Tel: +91-9637362911, Fax: +91-20-2553, E-mail: orthokunal@yahoo.com 


\section{Case Report}

A 24-year-old woman presented to our outpatient department with complains of severe mid back pain since 2 months. Patient was four months postpartum and lactating. The pain was so incapacitating that she was not able to sit or stand. There was no history of any recent or previous injury or fall, and no history of a fever or constitutional symptoms. She was of short stature, thinly built, and kyphotic posture. There was tenderness on palpation of lower dorsal and lumbar vertebrae and movements were painfully restricted. There was no sensory or motor deficit. There was difficulty in carrying out routine activities, which compelled the patient to seek advice.

Radiographs (Fig. 1) showed kyphoscoliosis and multiple vertebral fractures. An MRI of the spine (Fig. 2) showed vertebral compression fractures of D8, D9, D11, L2, and L3 vertebral bodies. Intervertebral discs and the spinal cord appeared to be normal. A detailed history was taken rule out cause of multiple pathological fractures. She was a multigravida (second delivery), having had her first child eight years ago. The first pregnancy was normal without similar complaints.

A complete metabolic workup was done which was normal except for a mild increase in serum alkaline phosphatase (160; normal range, 20-140 IU/L). A PET scan

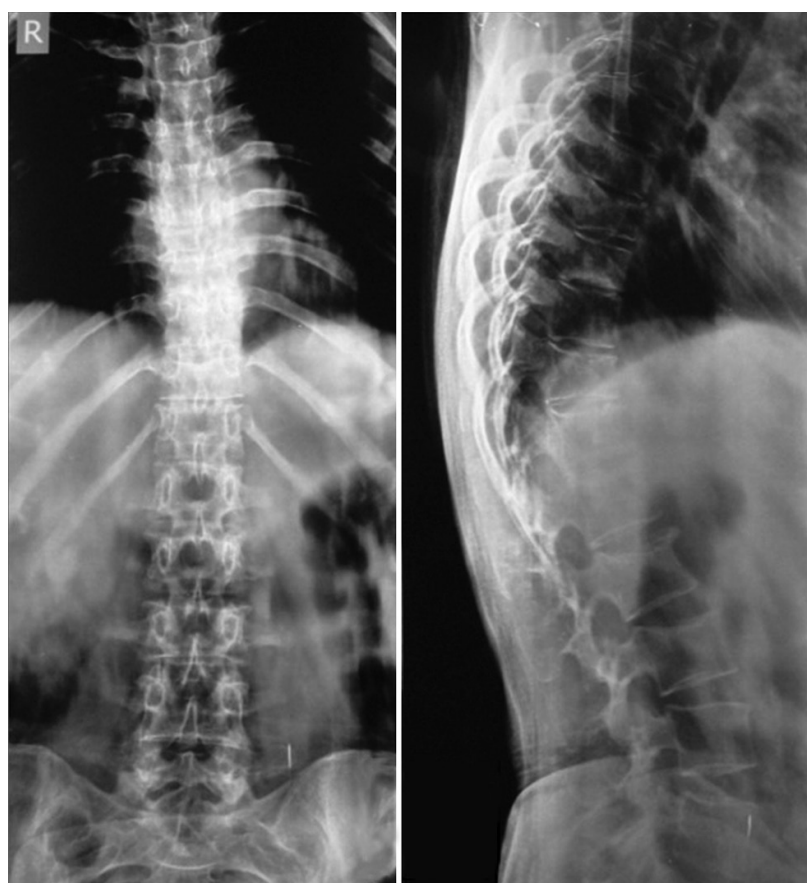

Fig. 1. Radiograph dorsolumbar spine anteroposterior and lateral view. was performed to rule out secondaries in view of raised alkaline phosphatase (Fig. 3), and it did not reveal any abnormalities. A dual energy X-ray absorptiometry (DEXA) scan of the spine and femur 45 region showed a $\mathrm{T}$ score of -4.5 at the lumbar spine and -2.8 at the hip region.

She was diagnosed with severe osteoporosis with multiple vertebral fractures and medical management and weaning was advised. She was reluctant to immediately wean her infant, so she was treated with total contact orthosis and vitamin $\mathrm{D}$ and calcium. At eight months postpartum, the pain subsided but DEXA scan showed no improvement in density scores. There were no new fractures detected on radiograph (Fig. 4). Lactation was stopped and she was started on teriparatide therapy along with vitamin $\mathrm{D}$ and calcium supplementation. At her one year follow-up, the patient was symptom free and DEXA scan showed improvement with $\mathrm{T}$ scores in the lumbar and hip region of -1.0 and -0.5 , respectively.

\section{Discussion}

Post pregnancy spinal osteoporosis is commonly seen during first three months of the postpartum period, especially after the first delivery. The symptoms include back pain which may progress to vertebral compression fractures and deformity [2]. The fractures are most commonly

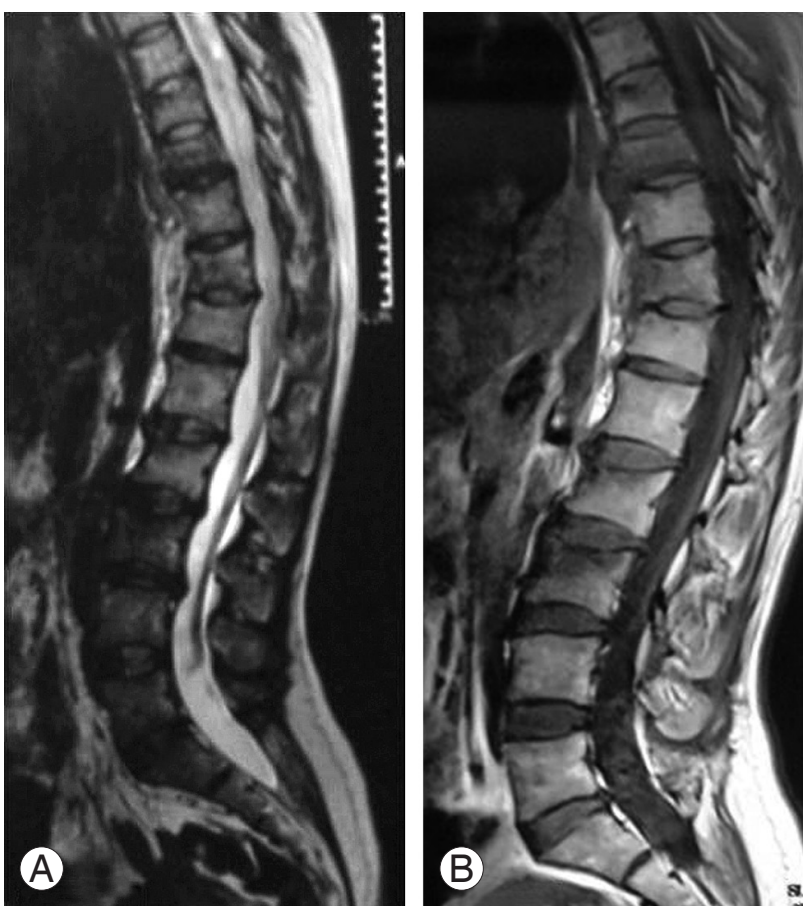

Fig. 2. (A) Sagittal T2 sequence. (B) Sagittal T1 sequence. 
seen in the lower thoracic and lumbar vertebrae [4].

The basic underlying pathological factor responsible for such severe bone loss during the postpartum and lactation phase is still not exactly clear. However there are certain risk factors like poor nutrition, low calcium intake, family history, smoking and corticosteroid intake which strongly increase the risk of PPSO [2,3]. Risk factors behind the pathophysiology of postpartum spinal osteoporosis include inadequate dietary intake of vitamin D and calcium, pre-existing disease, parathyroid hormone dysregulation, a weak form of osteogenesis imperfecta, anorexia nervosa, drug intake (corticosteroids or heparin), and bone loss during lactation [2]. Our patient did not have any of these risk factors.

Treatment described for postpartum spinal osteoporosis includes immediate cessation of lactation, calcium and

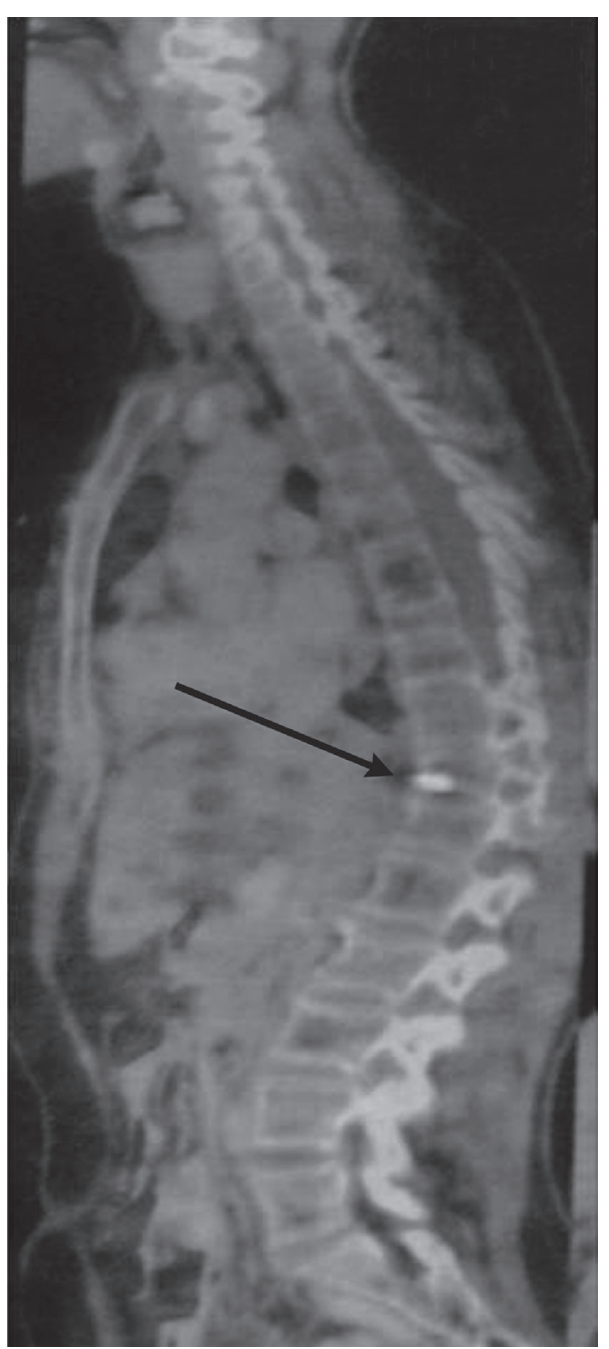

Fig. 3. Positron emission tomography-computed tomography scan, sagittal image showing hot spot. vitamin D supplementation, calcitonin, bisphosphonates, strontium ranelate and teriparatide [5-7]. Antiresorptives are the first line of management in postpartum osteoporosis with fractures [4]. Lactation results in the loss of 300-400 mg of calcium daily, which is compensated for by $5 \%-10 \%$ skeletal calcium loss. But this loss is gained back within few months of weaning [8]. Therefore weaning is advised as to treat PPSO. Calcium and vitamin D supplementation increase the levels of calcium in body and calcium absorption, respectively.

Although few reports have highlighted the use of bisphosphonates in the management of this condition, their safety in women of childbearing age has been questioned. Bone half-life of bisphosphonates has been shown to be around 10 years and has been reported to cause adverse effects during subsequent pregnancies and crosses the placenta, and forms deposits in the foetal skeleton [6]. Recent reports have shown good results with teriparatide, especially in women of childbearing age where bisphosphonates should be avoided $[4,6]$. Teriparatide is a human recombinant parathyroid hormone and an anabolic agent with half-life of only one hour, so cessation of therapy prior to future pregnancies does not cause any adverse reaction to the patient or foetus [4]. Although our patient was multigravida (second delivery), she wished to have
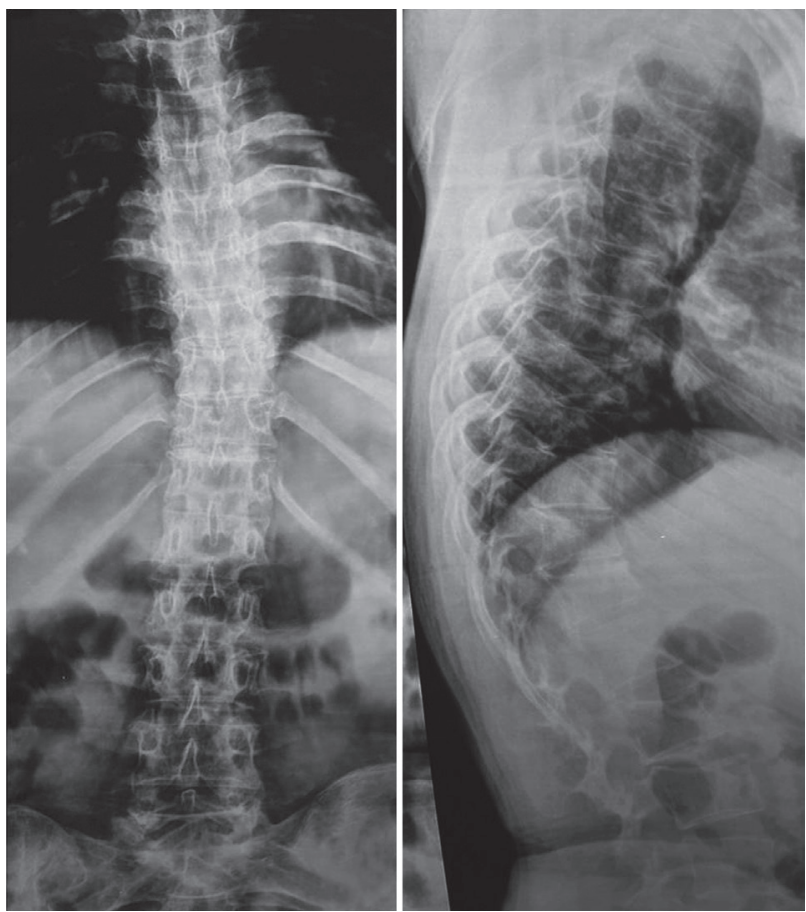

Fig. 4. Thoracolumbar spine radiograph in anteroposterior and lateral view at eight months postpartum. 
more children, therefore, we treated her with teriparatide. Good results have been observed with strontium ranelate, according to results from a few reports [5]. But because of its unknown mechanism and effects on skeleton, it is not commonly used. Moreover it is not approved by the food and drug association [4].

The World Health Organization recommends exclusively breast feeding for 4-6 months followed by weaning and introduction a supplemental diet. However in developing countries, breast feeding is commonly continued beyond 6 months and early weaning has significant social impact [9]. The majority of developing countries disagree with early weaning. So while treating PPSO, doctors need to keep in mind the safety profile of drugs during lactation. Although antiresorptives like bisphosphonates and teriparatide have been considered to be first line drugs in the management of PPSO, their safety during lactation is not proven [10]. Vitamin D and calcium can safely be used during the lactational period [10], but are less effective than antiresorptives as was seen in our case where no improvement in densitometry scores was observed. In this case, the mother was reluctant to wean; fortunately, she didn't develop any new fractures. Many patients wish to have multiple pregnancies and treatment with teriparatide is costly.

Many studies have pointed out need for setting up of controlled studies and guidelines for treating PPSO. We agree that a controlled study with the aim of setting guidelines for the treatment of this rare condition, while considering the socioeconomic factors is necessary.

In summary, our case presents a unique case of PPSO in a multigravida with a completely normal first pregnancy. PPSO is an important differential in any postpartum woman who presents with a complaint of back pain. Back pain and loss of height in a postpartum female should alert the physician to PPSO, especially if other risk factors are present. One needs to have high degree of suspicion to diagnose PPSO early, even in multigravida in order to prevent subsequent fracture and disability. Appropriate treatment and future counseling is of that utmost importance, while keeping the socioeconomic factors in mind.

\section{Conflict of Interest}

No potential conflict of interest relevant to this article was reported.

\section{References}

1. Dunne F, Walters B, Marshall T, Heath DA. Pregnancy associated osteoporosis. Clin Endocrinol 1993;39: 487-90.

2. Gomez de Tejada Romero MJ, Garcia Caballero A, Groba Marco M, Cardenes Leon A, Lazaro Archilla J, Sosa Henriquez M. Pregnancy-associated osteoporosis: presentation of 5 cases and long term monitoring. Rev Osteoporos Metab Miner 2012;4:57-62.

3. Di Gregorio S, Danilowicz K, Rubin Z, Mautalen C. Osteoporosis with vertebral fractures associated with pregnancy and lactation. Nutrition 2000;16:1052-5.

4. Lampropoulou-Adamidou K, Trovas G, Stathopoulos IP, Papaioannou NA. Case report: teriparatide treatment in a case of severe pregnancy- and lactationassociated osteoporosis. Hormones 2012;11:495-500.

5. Tanriover MD, Oz SG, Sozen T, Kilicarslan A, Guven GS. Pregnancy- and lactation-associated osteoporosis with severe vertebral deformities: can strontium ranelate be a new alternative for the treatment? Spine J 2009;9:e20-4.

6. O'Sullivan SM, Grey AB, Singh R, Reid IR. Bisphosphonates in pregnancy and lactation-associated osteoporosis. Osteoporos Int 2006;17:1008-12.

7. Choe EY, Song JE, Park KH, et al. Effect of teriparatide on pregnancy and lactation-associated osteoporosis with multiple vertebral fractures. J Bone Miner Metab 2012;30:596-601.

8. Pearson D, Kaur M, San P, Lawson N, Baker P, Hosking D. Recovery of pregnancy mediated bone loss during lactation. Bone 2004;34:570-8.

9. Caleyachetty A, Krishnaveni GV, Veena SR, et al. Breastfeeding duration, age of starting solids and high BMI risk and adiposity in Indian children. Matern Child Nutr 2013;9:199-216.

10. Friedman PA. Agents affecting mineral ion homeostasis and bone turnover. In: Brunton L, Chabner B, Knollman B, editors. Goodman \& Gilman's the pharmacological basis of therapeutics. 12th ed. New York: McGraw-Hill; 2011. p.1275-307. 